\title{
Switching points of view in spatial mental models
}

\author{
NANCY FRANKLIN \\ State University of New York, Stony Brook, New York \\ BARBARA TVERSKY \\ Stanford University, Stanford, California \\ and \\ VICKY COON \\ Georgia Institute of Technology, Atlanta, Georgia
}

\begin{abstract}
In six experiments, subjects read narratives describing varying spatial scenes with more than one point of view. They were probed with questions about objects located in six directions from each character's point of view. Subjects' response times were consistent with a one place-one perspective rule. They seemed to form separate mental models for separate places and to take a character's perspective when there was only one relevant character in a scene, but they seemed to take a neutral perspective when there was more than one probed point of view, rather than switch perspectives.
\end{abstract}

From discourse describing spatial situations, readers construct mental models of scenes in addition to mental representations of the language of the text (see, e.g., Bransford, Barclay, \& Franks, 1972; Bryant, Tversky, \& Franklin, 1992; Denis \& Cocude, 1989; Franklin \& Tversky, 1990; Glenberg, Meyer, \& Lindem, 1987; Mani \& Johnson-Laird, 1982; Morrow, Bower, \& Greenspan, 1989; Morrow, Greenspan, \& Bower, 1987; Perrig \& Kintsch, 1985; Taylor \& Tversky, 1992). Mental models contain information about the characters and objects in a scene, their orientations and locations. The spatial information preserved in them includes categorical spatial relations, such as those expressed in the words above, in front of, north of, across from, and so on, and sometimes it includes more analog information about distances. These mental models appear to be rapidly updated and transformed as narratives supply new information about objects, locations, and orientations. Experiments on the comprehension of spatial descriptions reveal processes underlying spatial thinking as well as those underlying language comprehension.

In many cases, readers' performance indicates that they have taken a specific perspective on a described scene (Abelson, 1979; Black, Turner, \& Bower, 1979; Bly, 1988; Bryant et al., 1992; Franklin \& Tversky, 1990; Glenberg et al., 1987). Switching perspective is timeconsuming and effortful (Black et al., 1979; de Vega,

This work was supported by AFOSR Grant 89-0076. We thank David Bryant, Keith Clayton, Michel Denis, Margaret Jean Intons-Peterson, Joe Kim, Jay Leahy, Nancy Stein, and one anonymous reviewer for their many helpful comments. Correspondence concerning this paper should be directed to N. Franklin, Department of Psychology, State University of New York, Stony Brook, NY 11794-2500.
1991), yet the comprehension of discourse sometimes requires it. The present experiments addressed how readers do that. Subjects read narratives that described spatial arrays around two characters, and they were queried about the locations of objects from each character's point of view, as each character changed orientation. To avoid confusion, we use point of view to refer to the viewpoints of characters in the described scenes, and perspective to refer to the viewpoints of readers of the described scenes. Readers' perspectives may or may not coincide with characters' points of view.

How might readers answer questions from each character's point of view? Previous research suggests that readers would take each character's perspective in turn. The spatial situations of the present experiments were based on those of Franklin and Tversky (1990) and Bryant et al. (1992). In the prototypical case (Franklin \& Tversky, 1990), subjects read narratives that described an observer oriented toward one object and surrounded by other objects beyond the observer's head, feet, back, left, and right. The narratives then described the observer facing each of the objects in turn, and the readers were queried for the objects in all directions from the observer. The readers apparently performed this task by adopting the point of view of the character, using a threedimensional mental scaffolding, termed a spatial framework (Franklin \& Tversky, 1990), to keep track of the objects as the character's orientation changed.

According to the spatial framework analysis (Franklin \& Tversky, 1990), readers construct a spatial mental model that consists of extensions of the three body axes, and they associate objects to it. They then transform the model as the narrative reorients the observer. The relative accessibility of the axes depends on characteristics 
of the body, characteristics of the perceptual world, and the relation of the observer to the perceptual world. This theory is based in part on analyses of spatial language by H. H. Clark (1973), Fillmore (1975), Garnham (1989), Levelt (1984), Miller and Johnson-Laird (1976), and Shepard and Hurwitz (1984). For an upright observer, the head/feet axis is most accessible because it is asymmetric and because it is correlated with gravity, the only asymmetric axis of the perceptual world. The front/back axis, though uncorrelated with a permanent perceptible axis of the world, has important behavioral and perceptual asymmetries. The right/left axis is not correlated with an axis of the world, nor does it have salient asymmetries, so it is the slowest of the three. In four experiments, when the observer was upright, response times to identify objects at specified directions followed the spatial framework pattern: times to the head/feet axis were fastest, followed by times to the front/back axis, and then by times to the right/left axis.

In subsequent experiments, the spatial situations and the observer's posture were varied, and the obtained patterns of data were consistent with variations of the spatial framework analysis. In some experiments, narratives described reclining observers who changed orientation by rolling from side to front to back to side (Franklin \& Tversky, 1990). When observers recline, no axis of the body is correlated with the gravitational axis, so the head/feet axis loses its primacy. The physical, perceptual, and behavioral asymmetries of the front/back axis loom larger than those of the head/feet axis, primarily because the perceptual and behavioral apparatus are oriented frontward. Thus, the spatial framework analysis predicts that for the reclining observer, the front/back axis should be fastest, followed by head/feet and then left/right. This pattern of data was obtained. Those experiments also served as a control for a confounding in the upright experiments. In the upright case, objects at head and feet never changed, and the head/feet axis was fastest. In the reclining case, as before, objects at head and feet were constant, but that axis was not fastest. It should also be noted that in several but not all of these experiments, subjects were fastest at the orientation (object faced) from which they had learned the scene. However, the same pattern of data emerged for all orientations.

The spatial framework analysis includes a theory of how perspective and orientation affect the retrieval of information in described scenes. In the original experiments, narratives described the observer as "you," to induce the reader to take the point of view of the observer. In subsequent studies, readers also took the points of view of an observer described in the third person, as well as that of an inanimate object, when they were questioned about other objects at specified directions from the third-person observer or object (Bryant et al., 1992). In those cases, the subjects could have taken the viewpoint of an outside observer, looking onto the other person or central object, but instead they took the viewpoint of the person or object. The spatial framework pattern of response times was also obtained in experiments in which subjects took the external viewpoint of an outside observer looking at another person surrounded by objects, when queried from the outside observer's point of view (Bryant et al., 1992). In the latter experiments, for some narratives, the scene was described from the point of view of the internal observer, and for other narratives, the scene was described from the point of view of an external observer. The slight differences in patterns of response times revealed that in both cases, the subjects took the internal or external point of view described in the narrative. Although there were two possible points of view, the subjects were queried from only one within a narrative; thus, in those experiments, they did not need to switch points of view.

In the experiments reported here, the subjects were required to respond alternately to two points of view within a description. They read narratives describing objects located with respect to two observers, or in one case, the same observer at two different times. They were queried with respect to each observer's point of view within the same narrative. The many previous experiments suggested that subjects would switch points of view by adopting the perspective of each of the observers in turn. If the subjects did this, a spatial framework pattern of response times should emerge for each observer, as in previous research (Bryant et al., 1992; Franklin \& Tversky, 1990).

To preview the present results, contrary to expectations, for all but one of the described situations involving two probed observers, the readers did not appear to take the points of view of the two observers in turn. Rather, the readers seemed to adopt neutral perspectives from which inferences about both points of view could be derived. There are several ways in which this could be done. One would be to adopt an external but oblique or overview perspective that would allow subjects to "view" both observers and all objects simultaneously in the three-dimensional scene. Another would be to use a more abstract perspective-free spatial model that would include both observers and all objects and allow computation of the probed spatial relations. Such a model would preserve the spatial relations among observers and objects much as a structural description preserves the spatial relations among the parts of an object (see, e.g., Marr \& Nishihara, 1978; Pinker, 1984; Ullman, 1989). As a whole, such a model would not be visualizable, but specific perspectives on it could be visualized. Taylor and Tversky (1992) found that readers formed perspective-free models of environments from reading either route or survey descriptions of them and used these general models to derive answers to perspective-laden questions.

If readers use perspective-free mental models or take oblique perspectives, there would be no reason to expect an upright spatial framework pattern of data. That pattern depends on readers' taking the perspective of characters in the scene and organizing the scene relative to their own body axes. So if readers have no particular perspective or a perspective oblique to that of the observer(s), the readers' body axes would not serve as a basis for a 
mental framework, and the spatial framework predictions about relative availability of axes would not apply. In this case, all directions should be equally available (Franklin \& Tversky, 1990; Levine, Jankovic, \& Palij, 1982). However, left and right frequently produce difficulties and slower response times in many different situations (see, e.g., Corballis \& Beale, 1976; Farrell, 1979; Maki \& Braine, 1985; Maki, Grandy, \& Hauge, 1979; Sholl \& Egeth, 1981), so it is possible that the head/feet and front/back axes will be equally available but that left and right may be slower due to confusion. Moreover, others have suggested that in order to make left-right (parity) judgments, people normally take the appropriate perspective (Cooper \& Shepard, 1975; Parsons, 1987a, 1987b). Thus, yet another possibility is that readers take an overview perspective, an oblique perspective, or no perspective except in making left/right judgments. For any of those cases, the only differences that might be expected are slower right/left times. We term this pattern of data weak equiavailability.

In these situations, then, readers seem to do one of two things. They can switch between smaller mental models, each of which includes only one observer and relevant objects, taking the observer's perspective within each one. Or they can use a more comprehensive mental model that includes both observers and all relevant objects, taking a neutral perspective that allows them to switch reference points. These two alternatives seem to preserve different kinds of cognitive economy. In the first alternative, readers form two perspective-laden mental models, each with a smaller number of objects and relations. They retrieve these smaller models one at a time but must switch between them. In the second alternative, readers can save themselves the effort of switching between different mental models by using a single integrated model containing more objects and relations and switching reference points within it. Thus, the cost of switching mental models can be compensated for by incurring the cost of a larger and more complex mental model.

The present series of experiments consisted of successive attempts to induce readers to take the points of view of each observer in turn in order to discover when readers take observers' perspectives and when they do not. The first manipulations derived from considerations of cognitive economy: biasing sampling of one observer's point of view, and increasing the size and complexity of the scene. In the later manipulations, the size and complexity of the scene were left constant, and the spatial and temporal structure of the scene were varied.

For most descriptions, not all information can be maintained simultaneously within a reader's focus of attention. Instead, some aspects of a narrative are foregrounded, by recency of mention or grammatical signaling devices (Delancey, 1982; Morrow, 1985a; van Dijk \& Kintsch, 1983; Wallace, 1982), by importance to the reader (de Vega, 1991; Morrow et al., 1989), or by spatial proximity to the current focus of attention in the scene (Glenberg et al., 1987). Foregrounded information is accessed more quickly than background information. In Experi- ments $1,2,3$, and 4, one of the two observers in each narrative received special emphasis. This should induce readers to foreground that observer, thus yielding shorter latencies for preparing to answer questions about that observer.

\section{EXPERIMENT 1 Same Place, Same Objects}

In the first experiment, narratives described two observers who were standing together in the same environment and were surrounded by nearly the same set of objects, but who were not necessarily facing in the same direction. This was done to minimize the memory load, or the size of the mental model needed to be kept in mind. In the narratives, the observers periodically and independently faced new objects. After each new orientation, the readers were queried about the directions of all objects with respect to each observer. If readers adopted the perspective of the observer queried in each question, the spatial framework pattern should emerge for each observer. In addition, because the narratives called one observer "you," and the other observer by a proper name, readers should foreground "you." If they did so, then preparing to answer a question about "you" should be faster than preparing to answer a question about the other observer, independently of the adopted perspective.

\section{Method}

Subjects. Eleven subjects ( 5 men and 6 women) participated for course credit in introductory psychology or for pay. None served in any other study.

Narratives. One practice and six experimental narratives were used. Each described two observers (one of whom was called "you," and one of whom was given a one-syllable common first name) standing together in the same scene but not necessarily facing the same object. Of the observers not labeled as "you," half were male and half were female, and each name appeared in only one scene.

Each narrative was written in two parts. In the first, which was printed on paper, a title and a list of the objects in the environment were presented, followed by a description of six objects located above, below, ahead, behind, left of, and right of each observer. For example, in one scene, you and Hank were on a Navy ship and were surrounded by an anchor, a bell, an antenna, a cannon, a lifeboat, and a flag. Other scenes were a hotel lobby, a museum, a construction site, an opera house, and a barn. The beginning of the barn description is given here as an example:

You and your cousin Ted are visiting a farm and have wandered
into the barn. You and Ted have climbed onto a small hay loft sev-
eral feet off the barn floor. The wooden slats that the loft is made
of are just wide enough for two people to stand on, but there is
plenty of room on all sides. Beyond your head, a straight ladder
hangs lengthwise from hooks in the barn ceiling. From the way it
hangs, it looks a bit like a jungle-gym. Beyond your feet is an over-
sized log. A crack runs the entire length of one side. Beyond your
back is a woven hammock. It is suspended from two parallel beams
that run across the barn. Beyond your left is Ted. Beyond your front,
a long pitchfork lies atop some crates. The wooden handle can barely
be seen through the dirt that has accumulated on it. Beyond your
right is a large barrel. The wood from which it is made is wet and
rotting. Beyond Ted's left, a loosely wound coil of rope droops from
a wood peg in the barn wall. It is thick enough to lift a heavy piece
of machinery onto the hayloft. Beyond Ted's front is the pitchfork. 
Beyond Ted's head is the ladder. Beyond Ted's right is you. Beyond Ted's back is the hammock. Beyond Ted's feet is the log.

Objects were chosen to be large so that they could be in one of the designated directions simultaneously for both observers. Within a scene, the sizes of objects and the distances between them were all approximately equal. The direction of an object was always given with respect to "your" point of view first.

The second portion of each narrative was written in blocks, each of which consisted of text interspersed with 12 ( 2 observers $\times 6$ directions) questions probing the directions of objects from observers. The first sentences of each block described the two observers as reorienting themselves in the scene, either continuing to face the same object or turning to face either of the other two objects around them on the horizontal plane. The other observer was always reoriented first. The observers never faced each other. A visual detail of the object currently to "your" front was then described, without explicitly mentioning "you" and referring to the object descriptively (e.g, "the container") rather than by name (e.g., "the barrel"). A filler sentence, referring to neither observer and neither point of view, followed. As an example, one of the barn reorientation descriptions, with supporting text, is given here:

You and Ted are both anxious to continue exploring the barn. Ted turns to face the hammock.

You turn to face the pitchfork.

Both the pointed end and the rounded tip of the handle stretch beyond the crates on which the tool lies.

One of the farmhands seems to be a bit lazy about returning things to their proper places.

Procedure. The subjects studied the first portion of the narrative for as long as they wished, until they knew the directions of all objects from both observers. They then returned the printed sheet to the experimenter and completed the remainder of the narrative on an IBM AT computer. Sentences appeared one at a time on the computer screen, and subjects read all sentences at their own pace, striking a key to advance to the next.

A question, presented in three parts, followed each filler sentence of the narrative. The first part designated the observer according to whose point of view the question was asked. The name of the other observer or the word "YOU" appeared in all capital letters on the computer screen. The subjects were instructed to press the space bar as soon as they felt that they were prepared to receive a question about the designated person. The time to press the space bar after the name appeared was RT1, or observer time. The observer's name then disappeared from the screen and was replaced by the second part of the question, which designated a direction from the specified observer ("HEAD," "FEET," "FRONT," "BACK," "RIGHT," or "LEFT"). The subjects were instructed to press the space bar as soon as they knew which object was in that direction from the specified observer. The time between the appearance of the direction label and the depression of the space bar was RT2, or direction time. The third part of the question consisted of a list of all six objects surrounding the designated observer from which the subject was to choose. The time to select an alternative from the list was RT3, or object time. (When both an observer and an object were in the same direction, the nearer of the two-that is, the observer-was given as a choice. The subjects were instructed about this.) The subjects were in structed to make all their responses as quickly as possible without sacrificing accuracy.

A new detail sentence, a filler sentence, and a question followed. After a block of 12 questions, the text reoriented both observers independently and continued with a new block of sentences and questions. Within each narrative, there were three blocks of questions.

Design. The order of stories was counterbalanced across subjects. The order in which the directions of objects were initially introduced, the serial positions of objects in the list of choices for questions, and the order in which the two observers faced the various objects were randomized. The order of questions in a block of 12 ( 2 observers $\times 6$ directions) was randomized, except that neither point of view was probed more than three times in a row.

Self-reports. After completing the set of narratives, the subjects were given a questionnaire in which they were asked whether they had experienced imagery during the experiment and what perspective(s) they took on the scenes. They were asked whether their perspective was that of an observer, a survey view, or some other perspective.

\section{Results}

Data were excluded from analyses for several reasons. Response times for questions answered incorrectly (2.1\% of response times) were eliminated. One subject's response times were eliminated for one narrative because he made more than $15 \%$ errors for that narrative. Very slow response times (more than 2.5 standard deviations from the observer $\times$ direction mean for a subject) constituted $4.7 \%$ of the data and were eliminated. Because variances were not equal across the various cells, a log transformation was conducted on all data prior to analyses in this and subsequent experiments.

Observer. RT1 data were subjected to a paired $t$ test comparing the times that subjects took to prepare for a question about the two observers. RT1 for "you" $(1.75 \mathrm{sec})$ was faster than it was for the other observer $(1.91 \mathrm{sec})[t(10)=2.63, p<.05]$. Observer was significant for RT2 + RT3 as well $\left[F(1,10)=7.47, M S_{\mathrm{e}}=\right.$ $.002, p<.05$ ], with the times for "you" $(3.25 \mathrm{sec})$ faster than those for the other observer $(3.47 \mathrm{sec})$. Observer times (RT1) for all experiments are reported in Table 1.

Dimension. The data for RT3 were analyzed in a 2 (observer) $\times 3$ (dimension) repeated measures analysis of variance (ANOVA). If subjects followed instructions and pressed the key indicating direction (RT2) only after they had retrieved the target object, RT3 would depend only on the serial order of the correct answer in the list of alternatives, which was randomized. Thus, if subjects were following instructions, there should be no effect of observer or dimension in RT3. Because spillover effects were found, both here $\left[F(2,20)=4.74, M S_{c}=.043\right.$, $p<.05]$ and in other experiments, we report results for the sum of RT2 and RT3. Means for the sum of RT2 and RT3 for all experiments are displayed in Table 1.

Because the predictions that we are comparing are made with respect to the head/feet, front/back, and right/left dimensions, we report comparisons of dimension and not direction. A 2 (observer) $\times 3$ (dimension) repeated measures ANOVA on RT2 + RT3 revealed significant effects for both dimension $\left[F(2,20)=6.28, M S_{\mathrm{e}}=.055\right.$, $p<.01]$ and the dimension $\times$ observer interaction $\left[F(2,20)=9.00, M S_{\mathrm{e}}=.091, p<.005\right]$. By Tukey's $H S D$ comparisons, using a significance level of .05 , right/left $(3.51 \mathrm{sec})$ was slower than both head/feet $(3.29 \mathrm{sec})$ and front/back $(3.29 \mathrm{sec})$, and head/feet and front/back did not differ. The same pattern was found for the other observer, with right/left $(3.84 \mathrm{sec})$ slower than 
Table 1

Mean Response Times for All Experiments

\begin{tabular}{|c|c|c|c|c|c|}
\hline \multirow[b]{2}{*}{ Observer (RT1) } & \multicolumn{5}{|c|}{ Dimension (RT2 + RT3) } \\
\hline & $\begin{array}{c}\text { Head/ } \\
\text { Feet } \\
(\mathbf{H})\end{array}$ & $\begin{array}{c}\text { Front/ } \\
\text { Back } \\
\text { (F) }\end{array}$ & $\begin{array}{l}\text { Right/ } \\
\text { Left } \\
\text { (R) }\end{array}$ & $M$ & Effects ${ }^{*}$ \\
\hline
\end{tabular}

Experiment 1: Same place, same objects, equal questions

$\begin{array}{lllllll}\text { You } & 1.75 & 3.29 & 3.28 & 3.17 & 3.25 & \mathbf{H}=\mathbf{F}=\mathbf{R} \\ \text { Other observer } & 1.91^{*} & 3.29 & 3.29 & 3.84 & 3.47^{*} & (\mathrm{H}=\mathbf{F})<\mathbf{R} \\ & & 3.29 & 3.28 & 3.50 \dagger & \end{array}$

Experiment 2: Same place, same objects, biased questions

$\begin{array}{lllllll}\text { You } & 2.06 & 2.76 & 2.99 & 3.75 & 3.17 & \mathrm{~F}=\mathrm{H}<\mathrm{R} \\ \text { Other observer } & 2.25 & 2.74 & 2.70 & 3.08 & 2.84 & \mathrm{H}=\mathrm{F}=\mathrm{R} \\ & & 2.75 & 2.84 & 3.41^{*} & \\ & & & & (\mathrm{H}=\mathrm{F})<\mathrm{R} & \end{array}$

Experiment 3: Same place, different objects

$\begin{array}{lllllll}\text { You } & 3.22 & 3.76 & 3.59 & 3.95 & 3.77 & (\mathrm{H}=\mathrm{F})<\mathrm{R} \\ \text { Other observer } & 3.61^{*} & 3.84 & 4.04 & 4.15 & 4.01^{*} & \mathrm{H}=\mathrm{F}=\mathrm{R} \\ & & 3.80 & 3.81 & 4.05^{*} & & \end{array}$

Experiment 4: Different places

$\begin{array}{lllllll}\text { You } & 3.02 & 3.44 & 3.80 & 4.35 & 3.86 & \mathrm{H}<\mathrm{F}<\mathbf{R} \\ \text { Other observer } & 3.28 & 3.55 & 4.18 & 4.60 & 4.11 & \mathrm{H}<\mathrm{F}<\mathbf{R} \\ & & 3.50 & 3.99 & 4.48 \ddagger & & \end{array}$

Experiment 5: One integrating place

\begin{tabular}{|c|c|c|c|c|c|c|}
\hline \multirow{3}{*}{$\begin{array}{l}\text { Observer } 1 \\
\text { Observer } 2\end{array}$} & 2.62 & 3.23 & 3.19 & 3.32 & 3.24 & $\mathbf{H}=\mathbf{F}=\mathbf{R}$ \\
\hline & 2.96 & 3.29 & 3.39 & 3.56 & 3.41 & $\mathbf{H}=\mathbf{F}=\mathbf{R}$ \\
\hline & & 3.26 & 3.29 & $\begin{array}{l}3.44 \\
H=F\end{array}$ & & \\
\hline
\end{tabular}

Experiment 6: Same place, one observer, two times

\begin{tabular}{lllllll} 
Earlier Time & 1.89 & 4.20 & 4.21 & 4.64 & 4.35 & $\mathrm{H}=\mathrm{F}=\mathrm{R}$ \\
Later time & $2.16 \dagger$ & 4.14 & 3.90 & 4.42 & 4.15 & $\mathrm{H}=\mathrm{F}=\mathrm{R}$ \\
& & 4.17 & 4.06 & 4.53 & 4.25 & \\
& & & \multicolumn{5}{c}{$\mathrm{H}=\mathrm{F}=\mathrm{R}$} \\
\end{tabular}

These effects interpret the main effect for the $H, F$, and $R$ entries of each row (condition); e.g., 3.29 vs. 3.28 vs. 3.17). They are based on post hoc tests. ${ }^{b}$ These effects interpret the main effect for the H, F, and R entries averaged over condition (e.g., 3.29 vs. 3.28 vs. 3.50 ). They are based on post hoc tests. ${ }^{*} p<.05$. $\dagger p<.01$. $\ddagger p<.001$.

both head/feet $(3.29 \mathrm{sec})$ and front/back $(3.29 \mathrm{sec})$ by Tukey's $H S D$ comparisons. No differences were obtained for "you."

Self-reports. All subjects reported experiencing imagery when reading the narratives and answering questions. Three of the 11 subjects reported consistently taking the perspective of one or both of the observers. Six subjects reported taking mainly a survey perspective, as if looking down on the observers and objects from above. Each of the other subjects said that they adopted different perspectives at different times. In all cases, there was no significant relation between self-report and individuals' response time patterns. This pattern is similar to that obtained in subsequent studies. Thus, self-reports will not be reported for the remaining experiments.

\section{Discussion}

Readers appeared to construct spatial mental models from the descriptions and use them to answer questions about objects located in various directions from observers. They easily and rapidly answered questions from new orientations, where the array of objects relative to the observers had to be inferred. Because the spatial framework pattern of response times did not appear, there was no indication that readers adopted the points of view of the two observers. Rather, the response times indicated that readers used more general mental models, including both observers, and took neutral perspectives. The only effect of dimension was that left/right was slower than the other dimensions, which is consistent with weak equiavailability. 
Of course, when some differences are not significant, the issue of power arises. This experiment was modeled on one that has produced differences many times over, but in a situation that differed critically. The actual differences between times for head/feet and times for front/ back were nil. Moreover, this pattern appeared in four additional experiments in this series and disappeared in a fifth, and an explanation for when differences did and did not occur will be provided.

The narratives gave precedence to "you" over the other observer. Preparing to respond to "you" took less time than did preparing for the other observer, and actual responses to "you" were faster than those to the other observer, indicating that readers foregrounded "you." This result is consistent with previous studies involving multiple characters, in which subjects foregrounded the character who was signaled by the text as primary or whose experience the text followed (de Vega, 1991; Morrow, 1985b; Stark, 1986).

\section{EXPERIMENT 2 \\ Same Place, Same Objects, Biased Questions}

In Experiment 1, readers seemed to adopt neutral perspectives rather than take the perspective of each observer in turn. They also foregrounded the observer called "you." The spatial situation in Experiment 2 was the same as that in Experiment 1, with one change: instead of equal numbers of questions for "you" and the other observer, $75 \%$ of the questions were about the other observer. This manipulation could have two effects. First, by emphasizing the other observer, it could induce readers to adopt that perspective. This would allow readers to use a spatial framework for organizing the space around that observer and for answering most of the questions. If subjects do adopt that perspective, a spatial framework pattern of response times should emerge for questions about the other observer. The second effect such a manipulation might have is to neutralize the foregrounding of "you" or even switch foregrounding of "you" to the other observer.

\section{Method}

Subjects. Ten subjects ( 4 men and 6 women) participated to fulfill an introductory psychology course requirement or for pay. None had served in Experiment 1.

Narratives and Procedure. The narratives and procedures were identical to those of Experiment 1, except that $25 \%$ of the questions (i.e., 3 questions within a block of 12) referred to "you" and $75 \%$ ( 9 questions per block) referred to the other observer. The subjects were not told that this would be the case, but before they began the experimental narratives they were given a practice narrative in which $75 \%$ of the questions referred to the other observer. Questions to be asked within a block were selected with the following constraints. (1) Across all stories, the set of questions for either point of view consisted of an equal number of questions about each direction. (2) All directions were probed for the other observer within each block.

\section{Results}

Unacceptable data were discarded according to the criteria used in Experiment 1. Errors constituted 3.9\% of the data. One narrative for each of 2 subjects and 2 narratives for each of 2 other subjects were discarded because the error rates for those narratives exceeded $15 \%$. Response times more than 2.5 standard deviations from a subject's observer $x$ direction mean constituted $6.0 \%$ of the data. Only the first instance of an observer $\times$ direction question within a block was included in the analyses.

Observer. A paired $t$ test did not reveal differences between RT1 for "you" $(2.06 \mathrm{sec})$ and the other observer $(2.25 \mathrm{sec})$. A second test, however, was conducted with only the last narrative completed by each subject. We reasoned that subjects realized by this time that the other observer was more likely to be probed, whereas they may not have realized this for earlier stories. In this second analysis, RT1 to the other observer $(2.05 \mathrm{sec})$ was significantly faster than to "you" $(2.45 \mathrm{sec})[t(9)=2.32$, $p<.05]$. A repeated measures ANOVA on RT2 + RT3 revealed no effect of observer, either for all stories or for only the last one.

Dimension. A 2 (observer) $\times 3$ (dimension) repeated measures ANOVA revealed an effect of dimension for $\mathrm{RT} 2+\mathrm{RT} 3\left[F(2,18)=4.93, M S_{\mathrm{e}}=.002, p<.05\right] . \mathrm{By}$ a Tukey's $H S D$ procedure, right/left $(3.42 \mathrm{sec})$ was found to be slower than both head/feet $(2.75 \mathrm{sec})$ and front/back $(2.85 \mathrm{sec})(p<.01)$. Front/back and head/feet did not differ from each other, and dimension did not interact with observer.

\section{Discussion}

Experiment 2, which emphasized the point of view of the other observer, was designed to provide circumstances more conducive to the use of a spatial framework than those in Experiment 1. Since three quarters of the probes asked about one of the observers, it seemed advantageous for readers to take that observer's perspective. This manipulation was ineffective. The spatial framework pattern of data did not emerge, even for the observer who was biased. The only effect of dimension was slower times for the right/left axis, which was consistent with weak equiavailability and indicative of use of a more general mental model.

Biasing the percentage of probes for the point of view of the other observer did neutralize the foregrounding of "you," and by the end of the experiment, it led to the foregrounding of the other observer. This apparently occurred because there were so many more questions for the other observer than for "you," and it occurred in spite of the fact that the descriptions emphasized "you."

\section{EXPERIMENT 3 Same Place, Different Objects}

Experiments 1 and 2 involved a relatively small number of surrounding objects. The readers did not seem to 
take the points of view of the two characters, even when one was probed three times as much as the other. Perhaps separating the observers in the scene and surrounding them by different sets of objects would induce the readers to use separate and smaller mental models to respond to direction probes. With two observers and 12 objects, a mental model representing all of the information at once would be rather large.

\section{Method}

Subjects. Thirteen subjects ( 5 men and 8 women) participated to partially fulfill a course requirement or for pay. None served in any other study.

Narratives and Procedure. The narratives and procedure were identical to those of Experiment 1, except that the two observers in each scene were described as being in separate parts of the same large scene (e.g., at different ends of a bam), and each observer was surrounded by a different set of six objects. For example, the two sets of six objects in the barn comprised a hammock, a log, a coil of rope, a pitchfork, a barrel, and a saddle, and a whiskey still, a nest, a bale of hay, a lawnmower, a basket, and a lantern. The objects were chosen to be about the same size and were randomly assigned to an observer and a direction.

\section{Results}

Unacceptable data were eliminated as in the previous experiments. Errors constituted $5.4 \%$ of the data, and $4.5 \%$ were eliminated because of very long response times.

Observer. For RT1 and RT2 + RT3, responses to questions related to "you" were faster than responses to questions related to the other observer. For RT1, "you" $(3.22 \mathrm{sec})<$ other $(3.61 \mathrm{sec})[t(12)=2.50, p<.05]$. For RT2+RT3, "you" (3.77 sec) < other $(4.04 \mathrm{sec})$ $\left[F(1,12)=6.25, \mathrm{MS}_{\mathrm{e}}=.006, p<.05\right]$.

Dimension. A dimension effect was obtained in RT2 +RT3 $\left[F(2,24)=4.44, M S_{e}=.008, p<.05\right]$. Tukey's $H S D$ analysis revealed that, overall, right/left $(4.05 \mathrm{sec})$ was slower than both head/feet $(3.80 \mathrm{sec})$ and front/back $(3.82 \mathrm{sec})$ at $p<.05$. Front/back and head/ feet did not differ from each other, and dimension and observer did not interact.

\section{Discussion}

Despite the fact that the narratives described observers in different parts of a scene and surrounded by different objects, so that a single model including both observers and all objects would be rather large, the readers seemed to use single comprehensive mental models and neutral perspectives rather than take the perspective of each observer in turn. The patterns of response times supported the predictions of weak equiavailability and not the spatial framework. As in the first experiment, readers also appeared to foreground "you."

\section{EXPERIMENT 4 Different Places}

Attempts to influence cognitive economy by biasing one observer and by increasing the size and complexity of the scene did not induce readers to take the separate perspectives of the two observers. A different kind of manipulation was in order. In Experiment 4, we changed the spatial descriptions by placing the two characters in different and unrelated places. The narratives described two observers in very different settings, such as a Navy ship and a hotel lobby, and each observer was surrounded by a different set of objects. Formally, the scenes in this experiment were identical to those in the previous experiment, with two observers and 12 objects. Psychologically, they were very different. Because the environments were described as physically distinct and physically separate, readers might establish a separate model for each environment.

\section{Method}

Subjects. Twelve subjects ( 6 men and 6 women) participated in partial fulfillment of a course requirement or for pay. None served in any other study.

Narratives and Procedure. Four narratives (describing a total of eight environments) were adapted from Franklin and Tversky (1990) and from Bryant et al. (1992) and were similar to those used in Experiment 1. The scenes within each narrative were chosen to be highly differentiable, and the observers were randomly paired with scenes. Below are parts of the initial description of the auditorium-and-barn narrative. The objects in the barn were a ladder, a hammock, a barrel, a pitchfork, a coil of rope, and a log. The objects in the auditorium were a set of blindfolds, a camera, a knife, a microphone, a pair of handcuffs, and a gag.

\begin{abstract}
You are an escape artist, and tonight you will invite several members of your audience to try to bind you in an inescapable position. Instead of bothering to learn the techniques of the masters. you have based your career on sneaky, cheap theatrics. You are standing in the middle of the stage, and you are making some last-minute checks on your props. Beyond your back is a pair of trick handeuffs lying open on a stool. You hope that tonight's volunteers decide to use them, since the fake metal teeth of the cuffs break easily with the slightest tug. Beyond your feet, you confirm that a knife built into the stage floor is concealed just below where you stand. The sharp blade is pointed up, enabling you to slice loose any ropes that will be tied around your ankles.

Ted is visiting a farm, and he has wandered into the barn. He has climbed onto a small hay loft several feet off the barn floor. The wooden slats that the loft is made of are just wide enough for one person to stand on, but there is plenty of room on all sides. Beyond Ted's left, a loosely wound coil of rope droops from a wood peg in the barn wall. It is thick enough to lift a heavy piece of machinery onto the hayloft.
\end{abstract}

Other pairs of scenes were a Navy ship and hotel lobby, a museum and lagoon, and an opera theater and construction site.

The descriptions and the questions were presented in the same way as were those for Experiment 1. The initial part of each narrative described the scene and objects surrounding "you," followed by the scene and objects surrounding the other observer. Detail sentences throughout the narrative described the object currently to "your" front

\section{Results}

Unacceptable data were discarded by using the same criteria as before. Errors constituted $5.4 \%$ of the data. The data from one narrative for each of 2 subjects were omitted because the error rates for those stories exceeded $15 \%$. All data from another subject were omitted because errors on all stories exceeded $15 \%$. This subject was 
replaced. Response times greater than 2.5 standard deviations above individual subjects' cell means constituted $5.4 \%$ of the data.

Observer. No observer effect was found for either RT1 or RT2+RT3. For RT1, the mean response times were $3.02 \mathrm{sec}$ to "you" and $3.28 \mathrm{sec}$ to the other observer. For RT2+RT3, mean response times were $3.86 \mathrm{sec}$ to "you" and $4.11 \mathrm{sec}$ to the other observer.

Dimension. For RT2 + RT3, dimension was significant $\left[F(2,22)=54.46, M S_{\mathrm{e}}=.015, p<.0001\right]$. Tukey's $H S D$ analyses revealed that head/feet $(3.50 \mathrm{sec})$ was faster than front/back $(3.99 \mathrm{sec})$, which was faster than right/left $(4.48 \mathrm{sec})$. The same pattern emerged for each of the observers separately, with all differences significant. Observer and dimension did not interact.

\section{Discussion}

Although previous attempts to manipulate factors of cognitive economy had not induced readers to take the respective perspectives of observers, describing the two observers as being in separate environments did. The spatial framework pattern of data emerged for both characters. In addition, neither observer was foregrounded.

Readers' behavior can be described somewhat speculatively as following a one place-one perspective rule. It has two parts. First, readers seem to use a single mental model containing all elements and spatial relations for a single described place, and separate models for separate places. Second, readers appear to adopt a single perspective for a single model. When a single place contains only one probed observer, readers take that observer's point of view. But when a single place contains more than one character or when more than one point of view is probed, readers take a neutral perspective, not that of either point of view. When readers assume a character's perspective, their mental models presumably contain only the objects associated with that observer; when they assume a neutral perspective, their mental models are more comprehensive, containing the objects associated with all observers.

Putting together these experiments with the previous ones, we can examine the effects of one or more observers in one or more places. In the earlier studies (Bryant et al., 1992; Franklin \& Tversky, 1990), there was one observer in one place, and the spatial framework pattern of data emerged, suggesting that subjects took the observer's perspective. In the present Experiments 1-3, there were two observers in a single place, and support for a neutral perspective was obtained. In Experiment 4, two observers appeared in two separate places, and evidence that readers took the points of view of the observers was obtained. The remaining case, that of a single observer in two places, has not been directly tested, though it seems likely that the pattern would be the same as that for two observers in two places.

\section{EXPERIMENT 5 One Integrating Place}

According to the results of Experiment 4, readers use one perspective for each place. This suggests that readers would use comprehensive mental models with a neutral perspective for the disparate environments of Experiment 4 if narratives integrated them by informing the readers that both could be viewed from the same place. For example, readers were told that they were standing on an oceanside cliff, with a view of both the Navy ship and the nearby hotel lobby. Thus, the narratives not only indicated that the two environments were contiguous but also provided the reader with a single survey perspective on the two observers in the two scenes. Neither observer was emphasized by the descriptions in this study.

\section{Method}

Subjects. Twelve subjects ( 5 men and 7 women) participated for course credit or for pay. None served in any other study.

Narratives. The pairs of environments used within each narrative in Experiment 4 were also used in Experiment 5. A different third-person observer was described in each environment, and "you" was always described as being in a position that allowed a survey view over both of the environments. The survey positions were on the top of a cliff, in a hovering helicopter, at the top of a telephone pole on a hillside, and on the observation deck of a tower. When one of the environments was indoors, a clear view was provided to the survey perspective (e.g., by having the hinged roof of the barn folded back for lifting heavy machinery with a crane). Sentences in the second portion of the narrative gave descriptive details of the visual and nonvisual sensory experiences from the survey perspective and did not favor either third-person observer.

\section{Results}

Unacceptable data were identified and eliminated as before. Errors constituted $3.8 \%$ of the data. Response times that were greater than 2.5 standard deviations above the subject's cell mean constituted $2.5 \%$ of the data. One narrative for 1 subject was eliminated because the error rate for that narrative exceeded $15 \%$.

Observer. No difference was found in RT1 between "you" $(2.62 \mathrm{sec})$ and the other observer $(2.96 \mathrm{sec})$. Similarly, "you" (3.24 sec) and the other observer $(3.41 \mathrm{sec})$ did not differ in RT2 + RT3.

Dimension. Dimension was nonsignificant in RT2+RT3, although the mean response time for right/left was the longest for both observers. Dimension did not interact with observer.

\section{Discussion}

Providing a single place from which the two disparate scenes could be viewed induced readers to use a single comprehensive mental model and a neutral perspective rather than to switch between models of each perspective or place, strengthening support for the one place-one per- 
spective rule. The pattern of data was consistent with equiavailability and not the spatial framework analysis; that is, there were no differences due to dimension, and, critically, there was no advantage of head/feet over front/ back. Furthermore, neither observer was foregrounded, which is consistent with their equal treatment by the text and task, neither observer was foregrounded.

\section{EXPERIMENT 6 \\ Same Place, One Observer, Two Times}

So far, the results are consistent with the one place-one perspective rule. Is there a comparable rule for time, a one time-one perspective rule? That is, will readers take the differing points of view of the same observer in the same place at different times? Or perhaps the one place-one perspective rule is so strong that readers will integrate the same observer in the same scene at different times into a single perspective, despite the impossibility of doing so in the "real" world.

\section{Method}

Subjects. Eleven subjects ( 7 men and 4 women) participated in order to partially fulfill a course requirement or for pay. None served in any other study.

Narratives and Procedure. The subjects read six narratives, each of which described a second-person observer ("you") in an environment at two different times of day. The observers were surrounded by six objects, and the objects faced at the two times were independent. In half of the narratives, the times were $1 \mathrm{~h}$ apart (e.g., 1:00 p.m. and 2:00 p.m.), and in half, they were $6 \mathrm{~h}$ apart (e.g., 1:00 p.m. and 7:00 p.m.). For each of these time discrepancies, half the stories described the earlier time first and half described the later first. The detail sentences all described features of the room as a whole that were true for both times (e.g., the stuffiness of the air).

\section{Results}

Of all the data, $3.6 \%$ were rejected as very slow (more than 2.5 standard deviations above the subject's cell mean), and $2.4 \%$ were errors.

Temporal point of view. The effect of temporal point of view was significant in RT1, with the earlier point of view $(1.89 \mathrm{sec})$ faster than the later point of view $(2.16 \mathrm{sec})[t(10)=4.03, p<.005]$. All 11 subjects showed this ordering of RT1 means (binomial probability $<.0005$ ). (Note that the terms earlier and later in Table 1 are used for the present purposes; these labels were not used in the narratives.) No effect of point of view was found in RT2 or RT2 +RT3. Separation of the two temporal points of view ( 1 vs. 6 h) did not produce a difference in response times. We therefore collapsed across this factor in subsequent analyses.

Dimension. Dimension was nonsignificant in RT2+RT3. Right/left, however, had the longest mean response time for both observers.

\section{Discussion}

The final experiment provided further support for the one place-one perspective rule and no indication at all that separate times and separate points of view lead to the use of separate perspectives. On the contrary, readers used a neutral perspective of the same observer in the same scene at two different times. The data fit the equiavailability or weak equiavailability pattern rather than the spatial framework pattern.

Another striking result of Experiment 6 is the differential accessibility of the two temporal points of view. Overall, and for all subjects, the point of view described in the narrative as being the earlier time of day produced shorter times, regardless of the order of mention in the text. Temporal cues have produced foregrounding effects in previous work as well. Stark (1986), for example, used the terms then and meanwhile to indicate the relevant scene of a multiple-scene narrative, and comprehension times for the ongoing narratives showed a foregrounding effect.

\section{GENERAL DISCUSSION}

\section{Foregrounding}

In two of the three cases in which description or task differentially emphasized one observer, that observer was foregrounded in the readers' mental models. The readers were faster to prepare to answer questions as well as to answer questions about the foregrounded observer. Typically, the foregrounded observer was emphasized in the narratives and was called "you" rather than by a proper name. There was one notable exception. For the case in which the other person's point of view was probed three times as often as "yours," the readers eventually seemed to foreground the other observer. Also notable was the case in which "you" was the observer at two different times. Although the times were emphasized equally and counterbalanced in order of presentation, the readers foregrounded the earlier time.

\section{Mental Models and Perspective}

Six experiments investigated readers' spatial mental models acquired from narratives and used to identify objects located with respect to two observers' changing points of view. In previous research on narratives that probed only one observer, readers were found to adopt the observer's point of view (Bryant et al., 1992; Franklin \& Tversky, 1990). In contrast, in the present research, for a variety of described situations that had more than one probed observer, the readers appeared to take neutral perspectives rather than those of each observer in turn. In order to do so, the readers appeared to use models that included all objects, observers, and relations, and that were perspective free or had an oblique perspective, rather than more limited models with an observer's perspective that included only that observer and the surrounding objects. Neither foregrounding one character (Experiments 1-3) nor asking three times as many questions from one character's point of view (Experiment 2) induced readers to take that character's point of view. Nor did increasing the size and complexity of the scene (Experiments 3 and 5 ) cause the readers to adopt the observers' perspectives. 
Although these variations in factors of cognitive economy did not affect the nature of mental models used by the readers, variations in the spatial settings described by the narratives did. When narratives described the two observers in disparate places rather than in different parts of the same place, even though the situations were otherwise equivalent, the readers seemed to use separate models of each place and to take the points of view of each observer in turn (Experiment 4).

This led us to propose a principle to account for the findings, the one place-one perspective rule. Readers appear to ground their mental models in a particular place, using one model for each place. In an entirely different paradigm, Radvansky and Zacks (1991) also found that place served as a basis for mental models. The one place-one perspective rule received further support from the final two experiments in the present series. When narratives described a single place from which both disparate environments could be viewed (Experiment 5), the readers used an integrated mental model and did not take the observers' points of view. When narratives described the same character in the same place at different times (Experiment 6), place again was the determining factor. The readers seemed to use models that integrated both time periods in a single place and did not take the character's perspectives, even though it was the same person at different times and orientations. Although different places led to different models, different times did not, despite the violation of physical reality.

Given the special status of place, what counts as a place? What the readers counted as a single, separate place seemed to derive from what the narratives described as a separate place. In the narratives, the different places were very different, typically in different buildings or different outdoor sites with different names and well-defined boundaries. They corresponded to different basic-level scenes (Tversky \& Hemenway, 1983) and contained objects from different and appropriate categories. In order to integrate two separate places, the narratives stated that they were contiguous and provided a third well-defined place from which both could be seen. Thus, the readers seemed to respond in a straightforward way to the narratives, using a separate model for each place that was described or implied to be separate.

For each place, the readers appeared to take a single perspective. When there was only a single point of view, as in Experiment 4 and in the prior research, the readers took the point of view of that character. In contrast, when more than one point of view was probed in a single place, as in the other experiments, the readers did not take the respective points of view of the observers. Rather, they appeared to use models that included all spatial relations and a neutral perspective from which all viewpoints could be derived.

Like place, perspective seemed to derive in a straightforward way from the narratives. When there were two characters in a single place, the narratives described both points of view and all objects as if they could be seen.
It seems natural, then, for readers to take a perspective that would allow both viewpoints and all objects to be perceived, or conceived. The readers appeared to do that, by using either a mental model with an oblique perspective or a more abstract perspective-free mental model.

Clearly, readers can use either type of model, perspective-laden and limited or perspective-neutral and comprehensive, and with ease. It is remarkable that for this range and type of situation, spatial characteristics of the described scene rather than general considerations of cognitive economy seem to determine whether readers use limited and perspective-laden or comprehensive and perspective-neutral mental models. This finding is not unprecedented, however. In research on solving geometric analogies, domain-specific spatial knowledge rather than general constraints on working memory determined order of performing transformations (Novick \& Tversky, 1987).

Place, but not time, served as a basis for the mental models. There may be several reasons why this happened. The mental models were used to keep track of six spatial directions under changing spatial orientation. Had the models been used to keep track of complex and changing temporal relations, time might have served as their basis of organization. However, there may be a deeper reason why place served as a basis for organizing mental models and time did not. Properties of the spatial world are reflected extensively in mental representations (Shepard, 1981). Knowledge of the spatial world, especially place, underlies people's ability to navigate in it, to describe it to others, and to comprehend such descriptions. Place is remembered remarkably well, even in incidental learning situations (see, e.g., Ellis, 1990; Hasher \& Zacks, 1979; Mandler, Seegmiller, \& Day, 1977; NavehBenjamin, 1988), so well that it easily serves as a basis for remembering other things, as in the method of loci. Psycholinguists have argued that our conceptions of time are based on those of space (e.g., H. H. Clark \& E. V. Clark, 1977). For example, temporal uses of terms like before and after derive from their spatial uses, and children grasp the temporal meanings of such terms only after they understand the comparable spatial meanings $(E$. V. Clark, 1972; H. H. Clark, 1973). Furthermore, adults provide better solutions to a problem presented as one of space allocation than to a formally identical problem presented as one of time allocation. The differences disappear when subjects solving the temporal analogue are given a hint to use a spatial representation (Carroll, Thomas, \& Malhotra, 1980). Part of the power of mental models comes from their ability to represent more abstract properties, such as time, spatially.

Although the one place-one perspective rule describes the present and past situations well, there are many situations that have not been explored, and that may limit the conclusions. For example, what if, in the same setting, one character were standing and the other reclining, or upside-down? Would the difficulty of calculating such disparate spatial relations induce readers to take the perspective of each observer? Or what if one point of view were 
that of an inanimate object, and the other that of a human body? Would readers be inclined to take the human's perspective? Yet another interesting case is that of a single observer in a single place, having flashbacks to a different time and place, where both present and remembered places would be probed in turn. And finally, does the tendency to take a neutral perspective when two points of view are probed in the same place apply to real as well as imagined scenes? Though not yet examined, this seems unlikely to occur in real life. That is, if one were actually in a situation requiring taking one's own point of view as well as that of another, it seems unlikely that a neutral perspective would be adopted instead of one's own. Although spatial mental models are derived from knowledge about the perceptual world, behavior in an imagined world and behavior in the real world do not always coincide (Bryant \& Tversky, 1991).

\section{REFERENCES}

ABELSON, R. (1979). Does a story understander need a point of view? In R. Schank \& B. L. Nash-Webber (Eds.), Theoretical issues in natural language processing (pp. 154-156). Washington, DC: Association for Computational Linguistics.

Black, J., Turner, T., * Bower, G. (1979). Point of view in narrative comprehension, memory, and production. Journal of Verbal Learning \& Verbal Behavior, 18, 187-198.

BLY, B. (1988). Perspective in mental models of text. Unpublished manuscript, Stanford University, Department of Psychology.

Bransford, J., Barclay, J., \& Franks, J. (1972). Sentence memory: A constructive versus interpretive approach. Cognitive Psychology, 3, 193-209.

Bryant, D. J., \& Tversky, B. (1991, November). Locating objects from memory or from sight. Paper presented at the meeting of the Psychonomic Society, San Francisco.

Bryant, D. J., Tyersky, B., FrankLin, N. (1992). Internal and external spatial frameworks for representing text. Journal of Memory \& Language, 31, 74-98.

Carroll, J. M., Thomas, J. C., \& Malhotra, A. (1980). Presentation and representation in design problem solving. British Journal of Psychology, 71, 143-153.

Clark, E. V. (1972). On the child's acquisition of antonyms in two semantic fields. Journal of Verbal Learning \& Verbal Behavior, 11, $750-758$.

CLARK, H. H. (1973). Space, time, semantics, and the child. In T. E. Moore (Ed.), Cognitive development and the acquisition of language (pp. 27-63). New York: Academic Press.

Clark, H. H., \& Clark, E. V. (1977). Psychology and language. New York: Harcourt Brace Jovanovich.

Cooper, L., Shepard, R. N. (1975). Mental transformations in the identification of left and right hands. Joumal of Experimental Psychology: Human Perception \& Performance, 104, 48-56.

Coranlus, M., Beale, I. (1976). The psychology of left and right. Hillsdale, NJ: Erlbaum.

Delancey, S. (1982). Aspect, transitivity and viewpoint. In P. J. Hopper (Ed.), Tense-aspect: Between semantics \& pragmatics (pp. 167183). Amsterdam: John Benjamins.

DENIS, M., CoCUDE, M. (1989). Scanning visual images generated from verbal descriptions. European Journal of Cognitive Psychology, 1, 293-307.

DE VEGA, M. (1991). Change of character and change of perspective in narratives describing spatial environments. Unpublished manuscript, University of La Laguna, Department of Psychology, La Laguna, Tenerife, Spain.

ELLIS, N. R. (1990). Is memory for spatial location automatically encoded? Memory \& Cognition, 18, 584-592.
FARRELL, W. S. (1979). Coding left and right. Journal of Experimental Psychology: Human Perception \& Performance, 5, 42-51.

Fillmore, C. J. (1975). Santa Cruz lectures on deixis. Bloomington: Indiana University Linguistics Club.

FrankLIN, N., TVersky, B. (1990). Searching imagined environments. Journal of Experimental Psychology: General, 119, 63-76.

Garnham. A. (1989). A unified theory of the meaning of some spatial relational terms. Cognition, 31, 45-60.

Glenberg, A., Meyer, M., Lindem, K. (1987). Mental models contribute to foregrounding during text comprehension. Joumal of Memory \& Language, 26, 69-83.

HASHER, L., ZACKs, R. T. (1979). Automatic and efforfful processes in memory. Joumal of Experimental Psychology, 108, 356-388.

LEVELT, W. I. M. (1984). Some perceptual limitations on talking about space. In A. J. van Doorn, W. A. van der Grind, \& J. J. Koenderink (Eds.), Limits on perception (pp. 323-358). Utrecht: VNU Science Press.

Levine, M., Jankovic, I., \& Palu, M. (1982). Principles of spatial problem solving. Journal of Experimental Psychology: General, 111, 157-175.

Maki, R. H. . Braine, L. G. (1985). The role of verbal labels in the judgment of orientation and location. Perception, 14, 67-80.

Maxi, R. H., Grandy, C., Hauge, G. (1979). Why is telling right from left more difficult than telling above from below? Journal of Experimental Psychology: Human Perception \& Performance, 5, 52-67.

Mandler, J. M. Seegmiller, D., \& Day, J. (1977). On the coding of spatial information. Memory \& Cognition, 5, 10-16.

Mani, K., \& Johnson-LaIRD, P. N. (1982). The mental representation of spatial descriptions. Memory \& Cognition, 10, 181-187.

MARR, D., Nishihara, H. K. (1978). Representation and recognition of the spatial organization of three-dimensional shapes. Proceedings of the Royal Society of London, 200, 269-291.

Miller, G. A., Johnson-LAiRd, P. N. (1976). Language and perception. Cambridge, MA: Harvard University Press.

Morrow, D. G. (1985a). Prepositions and verb aspect in narrative understanding. Journal of Memory \& Language, 24, 390-404.

Morrow, D. G. (1985b). Prominent characters and events organize narrative understanding. Joumal of Memory \& Language, 24, 304-319.

Morrow, D. G., Bower, G. H., GreenseaN, S. (1989). Updating situation models during narrative comprehension. Journal of Memory \& Language, 28, 292-312.

Morrow, D. G., Greenspan, S., Bower, G. H. (1987). Accessibility and situation models in narrative comprehension. Joumal of Memory \& Language, 26, 165-187.

Naveh-Benjamin, M. (1988). Recognition memory of spatial location information: Another failure to support automaticity. Memory \& Cognition, 16, 437-445.

Novick, L. R., Tversky, B. (1987). Cognitive constraints on ordering operations: The case of geometric analogies. Journal of Experimental Psychology: General, 116, 50-67.

PARSONS, L. (1987a). Imagined spatial transformation of one's body. Journal of Experimental Psychology: General, 116, 172-191.

Parsons, L. (1987b). Imagined spatial transformation of one's hands. Cognitive Psychology, 19, 178-241.

Perrig, W., Kintsch, W, (1985). Propositional and situational representation of text. Journal of Memory \& Language, 24, 503-518.

Pinker, S. (1984). Visual cognition: An introduction. Cognition, 18, 1-63.

Radvansky, G. A., Zacks, R. T. (1991). Mental models and fact retrieval. Joumal of Experimental Psychology: Leaming, Memory. \& Cognition, 17, 940-953.

ShePARD, R. N. (1981). Psychophysical complementarity. In M. Kubovy \& J. R. Pomerantz (Eds.), Perceptual organization (pp. 279-341). Hillsdale, NJ: Erlbaum.

ShEPARD, R. N., HURWITZ, S. (1984). Upward direction, mental rotation, and discrimination of left and right turns in maps. Cognition, 18, 161-194.

SHOLl, M. J., EGETH, H. E. (1981). Right-left confusion in the adult: A verbal labeling effect. Memory \& Cognition, 9, 339-350. 
STARK, H. (1986). Keeping track of characters in narrative. Unpublished doctoral dissertation, Stanford University.

TAYLOR, H. A., \& TVersky, B. (1992). Spatial mental models derived from survey and route descriptions. Journal of Memory \& Language, 31, 261-292.

Trersky, B., \& Hemenway, K. (1983). Categories of environmental scenes. Cognitive Psychology, 15, 121-149.

ULLMAN, S. (1989). Aligning pictorial descriptions: An approach to object recognition. Cognition, 32, 193-254.
Van DiJk, T., \& Kintsch, W. (1983). Strategies of discourse comprehension. New York: Academic Press.

WALLACE, S. (1982). Figure and group: The interrelationships of linguistic categories. In P. J. Hopper (Ed.), Tense-aspect: Between semantics \& pragmatics (pp. 201-223). Amsterdam: John Benjamins.

(Manuscript received August 15, 1991; revision accepted for publication April 13, 1992.)

\section{2nd Annual Meeting of the Society for Computers in Psychology \\ St. Louis, Missouri \\ November 12, 1992}

The 22nd Annual Meeting of the Society for Computers in Psychology will be held at The Adam's Mark Hotel in St. Louis on November 12, 1992, the day before the annual meeting of the Psychonomic Society. The meeting will include presentations, discussions, tutorials, and software and hardware demonstrations. The proceedings will be published in Behavior Research Methods, Instruments, \& Computers.

For further information regarding the conference, contact Peter Hornby or Margaret Anderson, Department of Psychology, SUNY/Plattsburgh, Plattsburgh, NY 12901 (phone, 518-561-3676; Bitnet, compsych @snyplava).

\section{3rd Annual Meeting of the Psychonomic Society \\ St. Louis, Missouri \\ November 13, 14, and 15, 1992}

The 33rd Annual Meeting of the Psychonomic Society will be held in St. Louis, November 13, 14, and 15, 1992. The meetings will begin Friday morning and continue until Sunday at noon. The headquarters hotel will be The Adam's Mark Hotel, at Fourth and Chestnut Street.

The program and hotel reservation cards have been mailed to members and associates. A copy of the program will be published in the November issue of the Bulletin of the Psychonomic Society. Additional programs wil be available at the registration desk for $\$ 7.00$.

For further information, please contact the secretary-treasurer of the Society: Cynthia H. Null. P.O. Box 7104, San Jose, California 95150-7104 (telephone: 415-604-1260). 